\title{
ON THE DYNAMICS OF SIMULTANEOUS SPREADING OF TWO-STRAIN DENGUE SEROTYPES
}

\author{
Leandro J. Vieira da Maia*, Kátya Regina de Freitas ${ }^{\dagger}$, Reginaldo A. Zara* $^{*}$ \\ * Universidade Estadual do Oeste do Paraná - Unioeste \\ Centro de Ciências Exatas e Tecnológicas \\ Rua Universitária, 2069 \\ Cascavel, PR, Brazil \\ † Universidade Tecnológica Federal do Paraná - UTFPR \\ Campus de Cornélio Procópio \\ Av. Alberto Carazzai, 1640 \\ Cornélio Procópio, PR, Brazil
}

Emails: leandro.jvmaia@gmail.com, kr_freitas@yahoo.com.br, reginaldo.zara@unioeste.br

\begin{abstract}
We discuss an epidemic model for dengue fever infection by considering the simultaneous spread of two serotypes throughout a shared non-naive host population and analyze the dynamics of the spreading for each serotype. The susceptible population is divided into two different susceptible classes: a naive population for those individuals that have never been exposed to the virus and a cross-imune population whose individuals have been exposed to one of the serotypes becoming immune to it but susceptible to its heterelogous. When the cross-immune populations are different an asymmetry in the susceptible host population is introduced and the dynamics of strains spreading is investigated as a function of this asymmetry. We show that the asymmetry in initial condition expressed by differences on the size of cross-imune population induces a feedback mechanism which produces an alternate pattern in epidemics with different dominant serotypes. We found that if the susceptible populations are symmetric both serotypes coexist during the epidemics but when the asymmetry becomes large two epidemics could be distinguished with a predominant serotypes in each one.
\end{abstract}

Keywords - Dengue Fever, Multiple Serotypes, Disease Spreading, Epidemiology

\section{Introduction}

Dengue fever is an infectious disease caused by viruses which could evolve to a severe infection and, depending on the form that it manifest itself could be classified as silent infection, dengue fever, dengue hemorrhagic fever, dengue shock syndrome. It is an important public health problem since this disease is endemic in more than 100 countries affecting more than 2.5 billion people living in tropical areas (Gluber, 2002; Feng and Velasco-Hernandez, 1997; Recker et al., 2009; Adams et al., 2006). The main transmitting agent are the mosquitoes Aedes aegypti and Aedes albopictus. The mean lifetime of the adult females is about 45 days and once infected by the virus they will remain infected until the end of their life. The transmission cycle occurs from an infected person to the susceptible mosquito which becomes infected and from infected mosquitoes to human susceptible individuals. The Dengue fever can be caused by each one of the four different virus serotypes known as DEN-I, DEN$I I, D E N-I I I$ and $D E N-I V$. Once a person is infected by one of the serotypes will never be reinfected by the same serotype again but will remain susceptible to the heterologous strain after a period of cross-immunity. Moreover and additional immunological response to reinfection known as antibody-dependent enhancement (ADE) which influences the susceptibility of the hosts to an heterologous serotype has also been pointed as an important aspect for reinfection patterns studies (Gluber, 2002; Recker et al., 2009; Wearing and Rohani, 2006).

The problem of multiple serotypes spreading in a population have been addressed in different ways. Nurani, Soewono and Sidarto (Nuraini et al., 2007) introduced the possibility of evolution to Dengue Haemorragic Fever in a deterministic model for two-strain serotypes. Derouich, Boutayeb and Twizell (Derouich et al., 2003) investigated a sucession of two epidemics caused by two different viruses using ordinary differential equations for hosts and vectors population. Esteva and Vargas (Esteva and Vargas, 2003) investigated a non-linear system of differential equations to model the coexistence of two serotypes in a given area taking into account the ADE effect expressing the idea that primary infection by a given serotype changes the probability of being infected by a heterologous serotype. They concentrated in the analysis of stability of the equilibruim points and established the conditions for the coexistence of two different serotypes in the same population based on the indirect interaction between these serotypes through the immunological memory expressed by the ADE. Chicaki and Ishikawa (Chicaki and Ishikawa, 2009) presented a dengue model considering sequential infections for different serotypes to simulate dengue transmission in Thailand. Their model takes into account sazonality effects in vector population, existence of unapparent cases, ADE and variability in 
the transmission probabilities for each serotype. The mathematical model was feed with empirical parameters obtained from the reports of the Queen Sirikit National Institute of Child Health (QSNICH) which summarized dengue fever occurrences in 1973-1999. They claim that all the four serotypes were found and that the dominant serotype change in time irregularly. By considering the relative frequency of serotypes occurrences they proposed that the dengue transmission probability ranges from $\lambda$ to $1.5 \lambda$, with $\lambda$ being the lower probability transmission from a vector to a host.

In a recent work Castanha et al (Castanha et al., 2013) performed a study about anti-dengue serotype-specific in a hyperendemic setting in northearstern of Brazil, using data on dengue reported cases collected during the year 2005. They found that number of exposed individuals to each one of the 3 circulating serotypes in Brazil are distributed in a non-uniform way. As a consequence, in a case of simultaneous circulation of different serotypes there will be a different set of available susceptibles to be infected an a different pattern of epidemics could emerge.

In empirical investigation is observed multiannual cycles in the disease incidence and different hypotheses to explain the alternance in the dominant serotypes have been discussed including ADE effects, heterogeneity in virus virulence, cross-immunity period and seazonal variation (Adams et al., 2006). However, none of them (solely or in combinational) provides a clear explanation of the observed empirical patterns. In this work we explore the observed heterogeneity in previously exposed population by simulating a twostrain spread of the disease in a non-naive host population and analyze the spreading dynamics for each serotype. Instead use a completely susceptible host population as an initial condition the population is divided in two different susceptible classes: a naive population for those individuals that have never been exposed to the virus and a cross-imune population whose individuals have been exposed to one of the serotypes becoming immune to it but susceptible to its heterelogous. Furthermore, the cross-immune populations may be different introducing an asymmetry in the susceptible host population, then the dynamics of spreading could be investigated as a function of this asymmetry. We show that the asymmetry in initial condition expressed by differences on the size of cross-imune population induces a feedback mechanism which produces an alternate pattern in epidemics with different dominant serotypes.

In the next section we present the compartmental model for simultaneuous two-strain serotypes spreading in a population including the cross-immunity state observed for dengue fever as a set of differential ordinary equations. We also propose a measure of the asymmetry for the initial conditions. In section 3 we present the results of the simulation for different asymetries and discuss the effect on the spreading dynamics.

\section{The mathematical model}

The mathematical model is based on the traditional susceptible-infectious-recovered classes for the human (host) and susceptible-infectious classes for the mosquito (vector). Let us consider the populations of hosts and vectors of sizes $H$ and $V$ and assume that the number of individuals of each specie is constant. Compartmental modeling divides the populations into categories or compartments according to their relative state of health and defines flow rates from a compartment to another taking into account the characteristics of the disease, the habitude of vector's transmission among other features. The host population is divided into classes being the most common the susceptible (S), infected (I) and removed ( $\mathrm{R}$ ) classes. For the dengue fever is observed that after recovering of a infection with given serotype a host becomes temporarily immune to all serotypes. Moreover, after a given period of immunity it becomes susceptible to the other serotypes. So two additional compartments related to the total and partial cross immunity should be included. The double-strain model is represented in Figure 1 by using a state flow diagram for the human host where the boxes represent the disease related stages and the arrows indicate the transition rates.

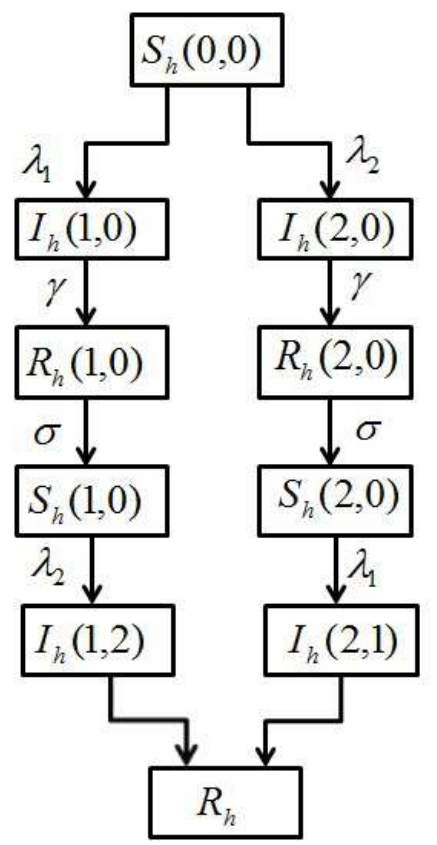

Figure 1: Transmission diagram for the two-strain epidemic model for human hosts.

To identify all the possible states each serotype is labelled as strain 1 and strain 2 and 
the states associated to the hosts have two indexes built with these labels $((i, j), i, j=0,1,2)$ where the label 0 is associated with no infection. The complete set of states for the host population is the following: $S_{h}(0,0)$ for naive individuals, $I_{h}(i, 0)$ for those infected by the strain $i$ only, $R_{h}(i, 0)$ for those recovered of infection of strain $i$ that are temporarily immune to all strains, $S_{h}(i, 0)$ for those who are imune to the strain $i$ only, $I_{h}(i, j)$ for hosts infected by the strain $j$ after being infected by strain $i$ and $R_{h}=\left(R_{h}(1,2)+\right.$ $\left.R_{h}(2,1)\right)$ for those are recovered from infections by strains 1 and 2 . For a closed populations, the constant size host population $H$ is divided into ten classes being $H=S(0,0)+I_{h}(1,0)+I_{h}(2,0)+$ $R_{h}(1,0)+R_{h}(2,0)+S_{h}(1,0)+S_{h}(2,0)+I_{(1,2)}+$ $I_{h}(2,1)+R_{h}$.

The ordinary differential equations for the two strain epidemiological model for a host population without death and births are given below and the dynamics of the model is described as follows. The symbols $I_{v}(i)$ set the population of infected vector with strain $i$ and $S_{v}(0)$ the susceptible vector population.

$$
\left\{\begin{aligned}
\frac{d S_{h}(0,0)}{d t}= & -\left(\lambda_{1} I_{v}(1)+\lambda_{2} I_{v}(2)\right) \frac{S_{h}(0,0)}{V} \\
\frac{d I_{h}(1,0)}{d t}=\lambda_{1} I_{v}(1) \frac{S_{h}(0,0)}{V}-\gamma I_{h}(1,0) & =\lambda_{2} I_{v}(2) \frac{S_{h}(0,0)}{V}-\gamma I_{h}(2,0) \\
\frac{d I_{h}(2,0)}{d t} & =\gamma I_{h}(1,0)-\delta R_{h}(1,0) \\
\frac{d R_{h}(1,0)}{d t} & =\gamma I_{h}(2,0)-\delta R_{h}(2,0) \\
\frac{d R_{h}(2,0)}{d t} & \sigma R_{h}(1,0)-\phi \lambda_{2} I_{v}(2) \frac{S_{h}(1,0)}{V} \\
\frac{d S_{h}(1,0)}{d t} & \sigma R_{h}(2,0)-\phi \lambda_{1} I_{v}(1) \frac{S_{h}(2,0)}{V} \\
\frac{d S_{h}(2,0)}{d t} & \phi \lambda_{2} I_{v}(2) \frac{S_{h}(1,0)}{V}-\gamma I_{h}(1,2) \\
\frac{d I_{h}(1,2)}{d t} & \phi \lambda_{1} I_{v}(1) \frac{S_{h}(2,0)}{V}-\gamma I_{h}(2,1) \\
\frac{d I_{h}(2,1)}{d t} & =\gamma\left(I_{h}(1,2)+I_{h}(2,1)\right) \\
\frac{d R_{h}}{d t} &
\end{aligned}\right.
$$

Susceptibles hosts $S_{h}(0,0)$ to both strains can be infected by the strain one or strain two with a rate $\left.\lambda_{i}\right)(i=1$ or 2$)$ when the infection is acquired by an individual in its first infection $\left(I_{h}(i, 0)\right)$. The infected hosts recover from this primary infection with a recovery rate $\gamma$ and get full and life-long immunity against the strain that they were exposed to, and also a short period of temporary cross-immunity against the other strain $\left(R_{h}(i, 0)\right)$. After the cross-immunity period they become susceptible to the second infection by a different strain. The susceptible host to the strain $j\left(S_{h}(i, 0)\right)$ can be infected with a rate $\lambda_{j}$ and gets the secondary infection. Then, the infected host $I_{h}(i, j)$ recovers with recovery rate $\gamma$ and become immune against all strains.

The vector individuals are assumed to be infected by only one serotype during its entire life. Moreover, as the mean life time of a vector is small it is assumed a birth and a death rate $\mu_{v}$ in order to keep the total population constant togheter a renewable number of susceptible individuals. This mean that infected vectors are removed from the system with a rate $\mu_{v}$ and new susceptible individuals are inserted at the same rate in order to satisfy the constraint $V=I_{v}(1)+I_{v}(2)+S_{v}(0)$.

Following the empirical observations of Chicaki (Chicaki and Ishikawa, 2009) and Castanha (Castanha et al., 2013) about the serotype frequency occurrences we assume an asymmetry in the initial susceptible host population and solve the model using as initial condition a naive population $S_{h}(0,0)<H$, and cross-immune populations $S_{h}(i, 0) \geq 0$ togheter an asymmetry expressed by the condition $S_{h}(1,0) \neq S_{h}(2,0)$.

To quantify the asymmetry let us consider a host population of size $H$ whose a fraction $f$ of individuals have been previously exposed to any of the strains 1 or 2 and those infected individuals are actually in the cross-immunity period. The host population is exposed again to both strains which could spread throughout the respective susceptible hosts. While a number of hosts $(1-f) H$ is susceptible to both serotypes a number $f H=S_{h}(1,0)+S_{h}(2,0)$ of hosts is susceptible to strain 1 or to strain 2 . Let us define an asymmetry factor in the susceptible population as

$$
\chi=\frac{\left|S_{h}(1,0)-S_{h}(2,0)\right|}{\left(S_{h}(1,0)+S_{h}(2,0)\right)} .
$$

If the number of susceptible individuals to each serotype are equal then $\chi=0$ and the total susceptible population is symmetric. On the other hand $\chi=1$ means that the population has been exposed to one of the serotypes, being completely susceptible to the heterologous one.

\section{Results and Discussion}

The model have been simulated for different values of asymmetry and infection rates $\lambda_{i}$. In all the simulations the infection rates $\lambda_{i}$ are the same for both strains but the ADE effect could be taken into account for the reinfection cases assuming $\phi>1.0$. Table 1 summarizes the complete set of parameters as well as the range they could assume in the simulation.

Initially the total population of vectors is assumed to be susceptible and a infection starts introducing a small number of human host individuals (chosen from the naive population) infected by each serotype. In this work it has been assumed an initial infection of 0.005 per cent of individuals 
Table 1: Range of parameters that may be used in the simulation.

\begin{tabular}{l|c|l}
\hline Parameter & Symbol & Value \\
\hline Host population & $H$ & $10^{5}$ \\
\hline Vector Population & $V$ & $10^{5}$ \\
\hline $\begin{array}{l}\text { Transmission rate Vector- } \\
\text { Host }\end{array}$ & $\lambda_{1,2}$ & {$[0,1]$} \\
\hline $\begin{array}{l}\text { Transmission rate Host- } \\
\text { Vector }\end{array}$ & $\lambda_{V}$ & {$[0,1]$} \\
\hline Recovering period & $\gamma^{-1}$ & {$[0,1]$} \\
\hline Cross immunity period & $\sigma^{-1}$ & {$[0,1]$} \\
\hline ADE parameter & $\phi$ & $\geq 1.0$ \\
\hline $\begin{array}{l}\text { Birth/death rate for Vector } \\
\text { population }\end{array}$ & $\mu_{v}$ & {$[0,1]$} \\
\hline
\end{tabular}

for each serotype, i.e., both strains could spread simultaneously throughout the available populations.

Figure 2 shows the fraction of hosts infected by any of the strains for $f=0.30, \lambda_{1}=0.20$, $\phi=1.0$ and different values of asymmetry factor $\chi$. Without loss of generality, for the asymmetric case it is assumed that $S_{h}(2,0) \geq S_{h}(1,0)$. For low asymmetry the system ehxibits the typical behaviuor of a SIR model since both strains spreads in the same way and the epidemics due to each particular serotype are indistinguishable. When the asymmetry factor is increased the behaviour is quite different since a secondary epidemic peak develops. The first peak is associated to a primary infection while secondary peak could be attributed to reinfection by a different strain.

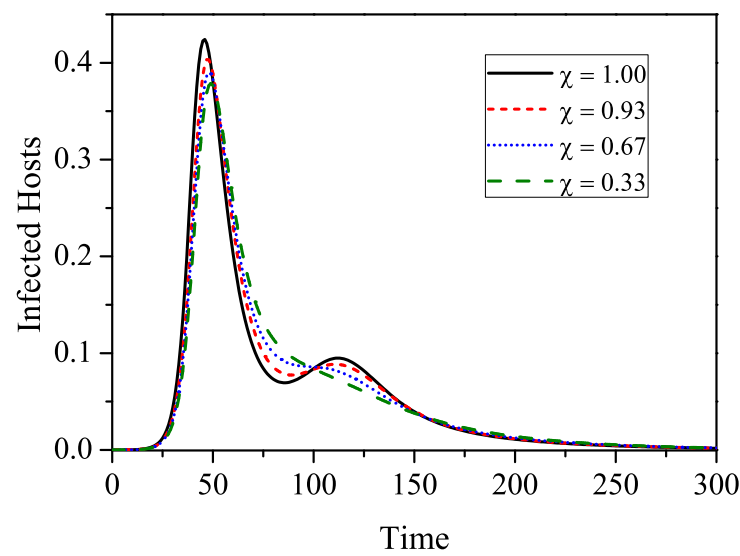

Figure 2: Fraction of infected hosts regardless the strain for different values of asymmetry $\chi$.

Although the Figure 2 provides an overview on the dynamics of the system a closer look at the details of the dynamics for each strain could be useful to understand the rising of the secondary peak and its relations with the asymmetry factor. In the Figure 3 the curves $I_{h}(i, j)$ correspond to the fraction of hosts infected by each serotype at each time step while the curve $I_{h}$ sets for the summation of infected individuals. For high asym- metry $(\chi=1.0)$, in the primary infection both strains spread simultaneously throughout a shared naive host population but the newly introduced serotype spreads also through the cross-immune population, i.e., the newly introduced serotype spreads over two populations generating a feedback process due the interaction between these population mediated by the transmission vectors. It could be seen at Fig.3(a) whose peaks of primary and secondary infection by serotype 1 have the same locus. Clearly the locus of primary infections taking place in the naive population should coincide since the force infection are the same for both serotype, but the number of infected hosts by serotype 2 is low since the available susceptible population is low. The infection period is followed by a cross immunity period in which the recovered population can not be infected by any strain. After the recovering of the primary infection by the strain $i$ and resting for a period as immune the individuals become available to infection by the strain $j$ and a secondary epidemics with the heterologous serotype takes place. In this case, a higher peak is observed for the serotype whose peak was low in the primary infection. When the asymmetry factor is lowered both strains spreads through two populations and the competition between the serotypes through the feedback process is continuously supressed, disappering for $\chi=0$.
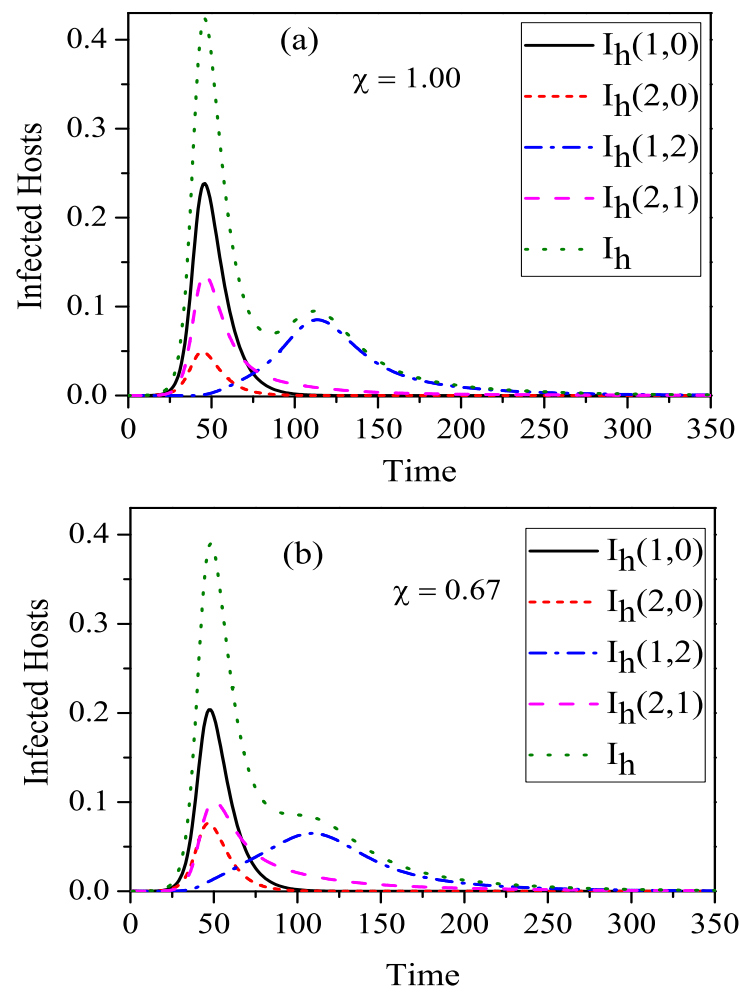

Figure 3: Infected hosts by serotype $i$ for different values of asymmetry $\chi$.

Let us analyze the feedback process taking place during the epidemics a little more closer. Figure 4 shows the fraction of susceptible popula- 
tion to strain $j\left(S_{h}(i, 0)\right)$. In this case the major part of cross-immune population is that previously exposed to strain $i=2$ being susceptible to strain $j=1$. At early times the fraction of susceptible population to strain 1 decreases quickly since the individuals are infected by strain 1 which spreads over populations $S_{h}(2,0)$ and $S_{h}(0,0)$, infecting the most of hosts and, as consequence, also the vectors. On the hand the strain 2 spreads over a small host population and the spreading is hampered by low number of available susceptible vectors, remaining in a small level in the system. For intermediary times the population infected by one serotype recovers and become susceptible to its heterologous. In this situation the population susceptible to strain 1 becomes larger than for strain 2 and a secondary epidemics takes place with a different dominant serotype.

It is clear in the Fig.3 that fraction of infected host vanishes for long time. In standard SIR models it happens since there are no hosts to be infected anymore. However in the case of two different serotypes spreading simultaneously, the competition between the strains give rise to an equilibruim point free of infection but with a nonvanishing fraction of suscetible individuals as illustrated in the Fig.4 and previosly investigated by Derouich,Boutayeb and Twizell (Derouich et al., 2003). In this figure could be seen that the majority of individual gets infected by the two strains during the epidemics, but a small fraction remains susceptible to a serotype and a new epidemics may develops depending on the level of susceptible population and the new exposition to the virus.

\section{Conclusion}

We discussed the dynamics of an epidemic model for dengue fever infection by considering the simultaneous spreading of two serotypes throughout a shared non-naive host population in which a cross-immune population gives rise to an asymmetry in the susceptible population. We have used an asymmetry in the initial condition expressed by the difference between cross-imune population which induces a feedback mechanism that gives rise to an alternate pattern in epidemics with different dominant serotypes at the primary and secondary epidemics. Moreover, it was found that if the susceptible populations are symmetric the serotypes coexist during the epidemics but if the asymmetry becomes large two epidemics could be distinguished with a predominant serotype in each one. Furthermore, when analyzing the multiannual alternance in the dominant serotypes during the epidemics the asysmmetry in the initial susceptible population should also taken into account together the seazonal, environmental and immunological features.
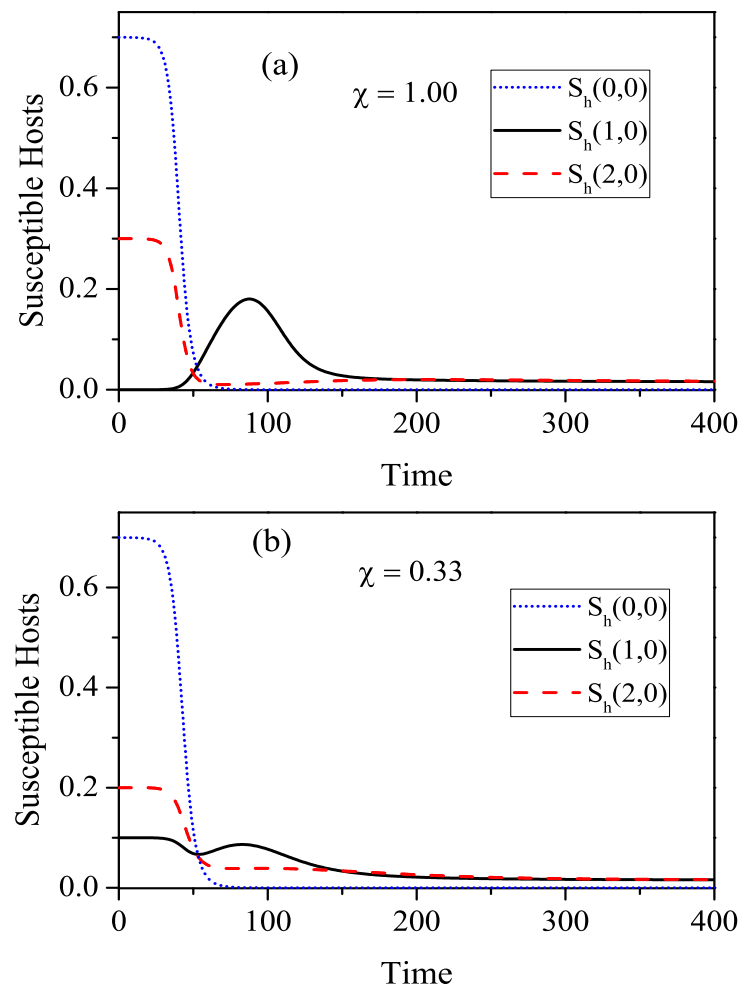

Figure 4: Susceptible hosts to serotype 1 $\left(S_{h}(1,0)\right)$, serotype $2\left(S_{h}(2,0)\right)$ and both serotypes $\left(S_{h}(0,0)\right)$ for different values of asymmetry in the initial condition.

Acknowledgements: The research was supported by Programa de Apoio a Núcleos e Excelência (Grant $N^{0} 550030 / 2010$ - 7, CNPq, PRONEX-Dengue - Brazil).

\section{References}

Adams, B., Holmes, E. C., Zhang, C., M. P. Mammen, J., Nimmannitya, S., Kalayanarooj, S. and Boots, M. (2006). Cross-protective immunity can account for the alternating epidemic pattern of dengue virus serotypes circulating in bangkok, PNAS 103: 1423414239. DOI: $10.1073 /$ pnas.0602768103

Castanha, P. M. S., Cordeiro, M. T., Martelli, C. M. T., Souza, W. V., Marques, E. T. A. and Braga, C. (2013). Force of infection od dengue serotypes in a population-based study in the notheast of brazil, Epidemiology and Infection 141: 1080-1088. DOI: $10.1017 /$ S0950268812001367

Chicaki, E. and Ishikawa, H. (2009). A dengue transmission model in thailand considering sequential infections with all four serotypes, J. Infec. Dev. Ctries 3: 711-722.

Derouich, M., Boutayeb, A. and Twizell, E. H. (2003). A model of dengue fever, BioMedical Engineering Online 2(4). 
Esteva, L. and Vargas, C. (2003). Coexistence of different serotypes of dengue virus, J. Math. Biol. 46: 31-37. DOI: 10.1007/s00285-002-0168-4

Feng, Z. and Velasco-Hernandez, J. X. (1997). Competitive exclusion in a vector-host model for the dengue fever, J. Math. Biol. 35: 523544. DOI: $10.1007 / \mathrm{s} 002850050064$

Gluber, D. (2002). Epidemic dengue/dengue hemorrhagic fever as public health, social and economic problem in the 21st century, Trends Microbiol. 10: 100-104. DOI: 10.1016/S0966-842X(01)02288-0

Nuraini, N., Soewono, E. and Sidarto, K. A. (2007). Mathematical model of dengue disease transmission with severe dhf compartment, Bull. Malays. Math. Sci. Soc. 30: 143157.

Recker, M., Blyuss, K. B., Simmons, C. P., Hien, T. T., Wills, B., Farrar, J. and Gupta, S. (2009). Immunological serotype interactions and their effect on the epidemiological pattern of dengue, Proc. R. Soc. B. 276: 25422548. DOI: $10.1098 /$ rspb.2009.0331

Wearing, H. J. and Rohani, P. (2006). Ecological and immunological determinants of dengue epidemics, PNAS 103: 11802-11807. DOI: 10.1073/pnas.0602960103 